\title{
Severe Pulmonary Embolism: Should Thrombolysis be Performed if the Clinical Probability is High Enough without an Echo-Heart?
}

\author{
Chebbah Houari* ${ }^{*}$ and A Boudahdir ${ }^{2}$ \\ ${ }^{1}$ Intensive Care Unit, Anesthesia Department, Public Hospital of Ain Defla, Algeria \\ ${ }^{2}$ Intensive Care Unit, Medico-Surgical Emergency Department, Blida University Hospital Center, Algeria
}

*Corresponding author: Chebbah Houari, Intensive Care Unit, Anesthesia Department, Public Hospital of Ain Defla, Algeria.

To Cite This Article: Chebbah Houari, Severe Pulmonary Embolism: Should Thrombolysis be Performed if the Clinical Probability is High Enough without an Echo-Heart?. Am J Biomed Sci \& Res. 2019 - 6(5). AJBSR.MS.ID.001076. DOI: 10.34297/AJBSR.2019.06.001076.

Received: 㘹 November 06, 2019; Published: 㘹 December 18, 2019

\begin{abstract}
Severe Pulmonary Embolism: Should thrombolysis be performed with high clinical probability and where echocardiography is unable to be practiced?

Background: Severe pulmonary embolism is considered as an urgent indication of intravenous thrombolysis.

Case presentation: Here we describe the case of an obese female patient of 34-year-old in the emergency unit, she had a typical clinical picture and recent history context that were very strongly suspicious of a serious pulmonary embolism, so the patient had undergone a successful intravenous thrombolysis (Quick and obvious recovery of the respiratory state) despite the fact that neither an echo-heart nor a Computed tomography angiography were able to be realized because patient transportation was impossible.
\end{abstract}

Discussion/Conclusion: In this case, the situation (when major complementary exams are unavailable or impossible to perform) adds more difficulties but such a heavy decision of acting thrombolysis or no remains possible. The right appreciation of the clinical context and surrounding factors could lead us to an accurate diagnosis. Computed tomography, Echo-heart, arterial Gasometer, remain major advancements of today's medicine and should not be ignored if available. Thrombolysis should be done with extra-carefulness and with a very fine evaluation of contraindications.

\section{Introduction}

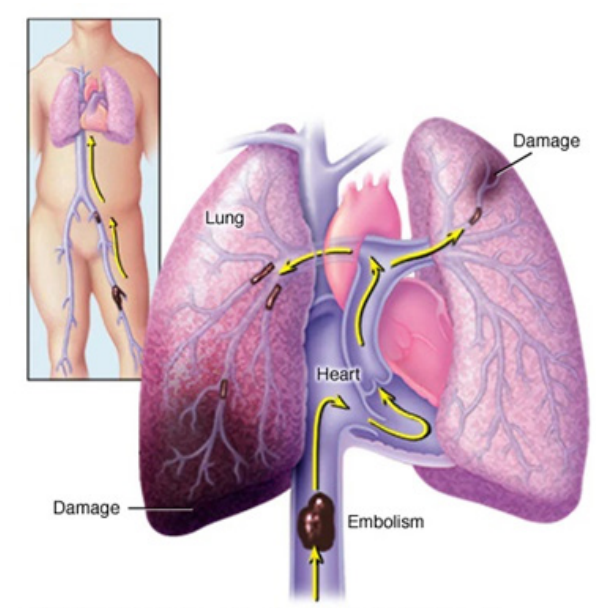

Figure 1: Embolism migration representation. 
Pulmonary Embolism (PE) could be manifested by different range of clinical presentation from asymptomatic to death, however in many cases we could have clinical signs that must not be ignored or underestimated (Figure 1). We describe here a case of a patient that has clinical context and recent history which give us a direct inquiry to PE.

\section{Case Presentation}

Female Patient, 34, no history, obese +++ BMI: 54, admitted to the emergency department for severe acute respiratory distress. The admission examination finds a patient conscious, very agitated, polypnea at $38 \mathrm{c} / \mathrm{min}$ with shallow breathing, no cough, rales are absent, well perceived VM, perioral cyanosis and coldness of the extremities. Spo2 30-40\%, BP: 70/30, HR: 140 $\mathrm{b} / \mathrm{min}, \mathrm{T}^{\circ} 37 \mathrm{c}$, pulse thready but very fast, no turgescence of the jugular, abdominal examination is without abnormalities. The patient's husband Interrogation finds the notion of a sprained left knee for which she benefited of a plastered splint without any preventive anticoagulation. The husband describes that the respiratory symptomatology set in abruptly manner. The rest of the examination finds a lower left slightly oedematized limb (ankle), with a significant decrease in the bale of the thigh and leg with a sensation of spontaneous contracture. The right lower limb is without defects. No sense of amenorrhea, no abdominal pain. EKG: sinus tachycardia. Blood sugar strips: no hypoglycemia. A biological assessment (Blood Count Formula, Prothrombin Time, Activated Partial Thromboplastin Time, Urea, creatinine, natremia, kalemia) does not find any defects. Chest X-ray in the patient's bed is unavailable.

\section{Wells score: 9, above 7: high probability.}

a) Heart rate $>100 \mathrm{~b} / \mathrm{min}$ : 1.5, Recent surgery or Recent surgery or immobilization: 1.5

b) DVT sign: 3, Diagnosis other than less likely than PE: 3

\section{Geneva score: 12 points $>$ 9: high clinical probability of PE.}

a) Surgery or recent immobilization $<1$ month: 3 points, Clinical signs DVT (painful palpation and edema): 4 points, Heart rate $>95 / \mathrm{min}: 5$ points.

In front of this picture, we thought we were very likely face to a pulmonary embolism. The combination of the shock board with a high clinical probability of a PE indicates the realization of a trans-thoracic echo-heart in the context of the emergency because the patient is not transportable for a computed tomography angiography chest-scan.

\section{Other likely diagnoses}

a) Shock State: hemorrhagic, anaphylactic, cardiogenic and septic.

b) Acute Respiratory Failure: No cardiologist on call, no ultrasound machine.

\section{Immediate Treatments (Conduct to Hold)}

Oxygen therapy, large peripheral pathway for vascular filling. Thirty (30) minutes later, the patient's condition deteriorated with worsening respiratory status, Spo2 at 50\% with high nasal oxygen flow, impregnable BP and signs of shock. The patient was placed on vasoactive drugs: Noradrénaline $®$, with no improvement despite aggressive doses of Noradrénaline®. Face to this table, a decision on thrombolysis was taken after eliminating the contraindications; Métalyse ${ }^{\circledR}$ 10,000 IU injected into IVD. (Directly in Venous). One hour after thrombolysis injection end, ithe improvement in respiratory status is spectacular; eupneic patient, the rales disappeared, the respiration became calm, an obvious cutaneous recoloration and disappearance of cyanosis, BP of 110/60 under Noradrénaline ${ }^{\circledR} 2 \mathrm{mg} / \mathrm{h}$, HR $110 \mathrm{~b} / \mathrm{min}$ Rapid withdrawal of Noradrénaline $\AA$. The next day, the patient was completely weaned from oxygen therapy. A heart echo was done and did not find any abnormalities, the same thing for the EKG which was strictly normal. Echo-doppler of the lower limbs founds a left ilio-femoral proximal deep vein thrombosis. The patient was placed on Enoxaparin ${ }^{\circledR}$ $0.8 \mathrm{ml} / 12$ hours sc. (Under Skin), with relay by oral Anti-vitamin K from the 4th day with a good evolution.

\section{Discussion}

A fine practitioner can deliver the best appreciation of the context and clinical exam together. In this case, the situation (when major complementary exams are unavailable or impossible to perform) adds more difficulties but such a heavy decision of acting thrombolysis or no remains possible. According to "ESC 2014" [1] and even "ESC 2019" [2] recommendation's, a Computed chest angio-scan is required in case of unstable but transportable patient. Otherwise if the patient is not transportable, an echo-heart is required. Echo-heart and vascular echo-Doppler formation are now a "must-have" for emergency physicians and anesthetists. The interest is also bigger for more skilled trainees. Preventive anticoagulation is more and more a "must be done" and not to be oversighted by all practitioners and was clearly missing in this case.

\section{Conclusion}

Pulmonary Embolism is a distress that could mean to death if adequate measures are not taken quickly.

The right appreciation of the clinical context and surrounding factors could lead us to accurate diagnosis.

Computed tomography, Echo-heart, arterial Gasometer, remain major advancements of today's medicine and should not be ignored if available. Thrombolysis should be done with extra-carefulness and with very fine evaluation of contraindications.

\section{References}

1. Stavros V Konstantinides, Adam Torbicki, Giancarlo Agnelli, Nicolas Danchin, David Fitzmaurice, et al. (2014) ESC Guidelines on the diagnosis and management of acute pulmonary embolism: The Task Force for the Diagnosis and Management of Acute Pulmonary Embolism 
of the European Society of Cardiology (ESC). European Heart Journal 35(43): 3033-3080.

2. Stavros V Konstantinides, Guy Meyer, Cecilia Becattini, Héctor Bueno, Geert-Jan Geersing, et al. (2019) ESC Guidelines for the diagnosis and management of acute pulmonary embolism developed in collaboration with the European Respiratory Society (ERS): The Task Force for the diagnosis and management of acute pulmonary embolism of the European Society of Cardiology (ESC). European Heart Journal p. 1-61. 